\title{
Progress in search for antihelium with BESS
}

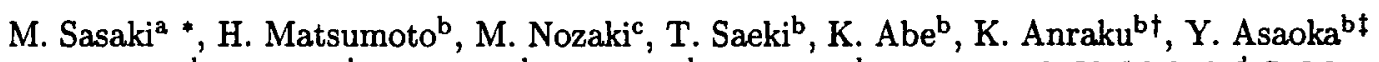

M. Fujikawa ${ }^{b}$, H. Fuke ${ }^{b}$, M. Imori ${ }^{b}$, S. Haino ${ }^{b}$, K. Izumi ${ }^{b}$, T. Maeno ${ }^{c}$, Y. Makida ${ }^{d}$ S. Matsuda ${ }^{b}$, N. Matsui ${ }^{b}$, T. Matsukawa ${ }^{c}$, H. Matsunaga ${ }^{b \S}$, J. W. Mitchell ${ }^{\mathrm{a}}$, T. Mitsui ${ }^{\mathrm{b}} \mathbf{9}$ A. Moiseev ${ }^{\mathrm{a}}$, M. Motoki ${ }^{\mathrm{b} \|}$, J. Nishimura ${ }^{b}$, S. Orito ${ }^{b * *}$, J. F. Ormes ${ }^{a}$, T. Sanuki ${ }^{b}$, Y. Shikaze ${ }^{b}$, E. S. Seo ${ }^{e}$, T. Sonoda $^{b}$, R. Streitmatter ${ }^{a}$, J. Suzuki ${ }^{d}$, K. Tanakad ${ }^{d}$, K. Tanizakic, I. Ueda ${ }^{b}$, J. Z. Wange, Y. Yajima ${ }^{f}$

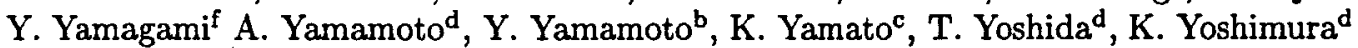

a National Aeronautics and Space Administration, Goddard Space Flight Center (NASA/GSFC), Greenbelt, MD 20771, USA

${ }^{b}$ The University of Tokyo, Tokyo, 113-0033 JAPAN

${ }^{c}$ Kobe University, Kobe, Hyogo, 657-8501 JAPAN

${ }^{d}$ High Energy Accelerator Research Organization (KEK), Tsukuba, Ibaraki 305-0801, JAPAN

eUniversity of Maryland, College Park, MD 20742, USA

${ }^{f}$ The.Institute of Space and Astronautical Science (ISAS), Sagamihara, Kanagawa 229-8510, JAPAN

We have searched for antihelium nuclei in cosmic rays using the data obtained from balloon flights of the BESS magnetic spectrometer. The search was mainly based on track-quality selection, followed by rigidity analysis, and on the time-of-flight and $\mathrm{d} E / \mathrm{d} x$ measurements by the scintillation counter hodoscope. We analysed all the data collected during 1993-2000 with a common analysis procedure. No antihelium nuclei events were found in the energy range from 1 to $14 \mathrm{GV}$. In order to determine a new upper limit, we have simulated the loss in the air and in the instrument of $\mathrm{He}(\overline{\mathrm{He}})$ using the GEANT/GHEISHA code. Combined with the data collected in 1993 through 2000, a new $95 \%$ confidence upper limit for the ratio of $\overline{\mathrm{He}} / \mathrm{He}$ at the top of the atmosphere of $6.8 \times 10^{-7}$ has been obtained to be after correcting for the interactions in the air and in the instruments.

\section{Introduction}

Continuous effort has been made to detect antiparticles in cosmic rays ever since Dirac first predicted the existence of antimatter. Now, it has been well established that there is a small

\footnotetext{
*NAS/NRC Research Associate

tCurrently at Univ. of Kanagawa, Kanagawa, Yokohama 221-8686, Japan

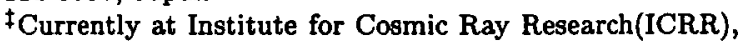
Univ. of Tokyo, Kashiwa, Chiba 277-8582, Japan

\$Currently at Univ. of Tsukuba, Tsukuba, Ibaraki 3058571, Japan

TCurrently at Tohoku University, Sendai, Miyagi 9808578, Japan

"Currently at Tohoku University, Sendai, Miyagi 9808578, Japan

**deceased
}

fraction of antiprotons $\left(\overline{\mathrm{p}} / \mathrm{p} \sim 10^{-4}\right)$ in cosmic rays, but yet no evidence that antimatter with $|Z| \geq 2$ exists. [1-3]. As the origin of cosmicray antiprotons is generally thought to be production in energetic collisions of Galactic cosmic rays with the interstellar medium, this result shows that our galaxy is made of matter and the local baryon asymmetry is maximal. The question is whether this asymmetry is also global in the universe. The absence of annihilation $\gamma$-ray peaks shows that little antimatter is to be found within $\sim 20 \mathrm{Mpc}$. However, the possibility of existence of antimatter clusters in the universe is not completely precluded. If one antihelium was observed in cosmic rays, it would be a strong indication of the existence of antimatter clusters because the 
probability of producing antihelium in collisions of cosmic rays with the interstellar medium is vanishingly small. The search for antihelium in the cosmic rays using the BESS detector was carried out, and a model-independent upper limit on the flux ratio $\overline{\mathrm{He}} / \mathrm{He}<3.1 \times 10^{-6}$ was achieved in previous analysis[2]. From 1993 to 2000, we have had seven successful flights with improvements in the detector performance year by year. In order to deal with the data for every year in a similar way, we re-analysed all the data collected during the period of 1993-2000 with a common analysis procedure. We have also simulated the loss of $\mathrm{He}(\overline{\mathrm{He}})$ in the air and in the instrument using the GEANT/GHEISHA code. Using these advances, we report here updated results.

\section{Instrument}

The BESS detector has a wide-open geometry with a large acceptance of $0.3 \mathrm{~m}^{2} \mathrm{sr}$. Details of the detector have been reported elsewhere [10-13].

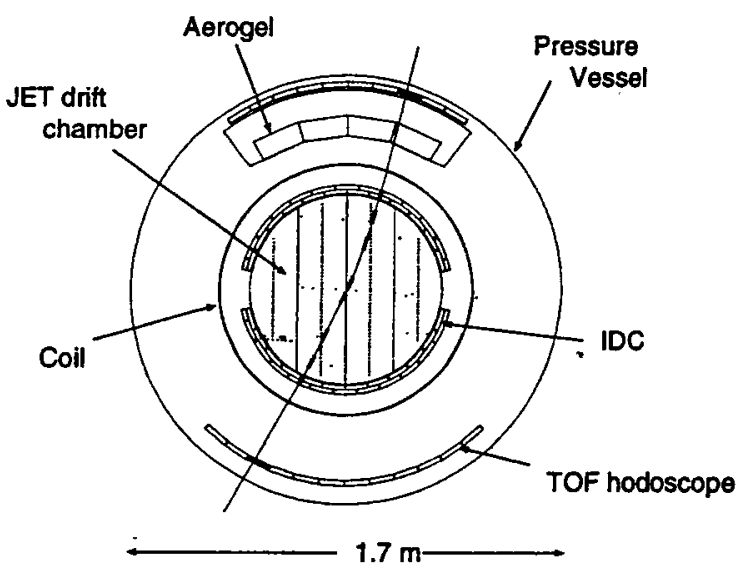

Figure 1. Cross-sectional view of the BESS spectrometer in its ' 97 configuration.

All the detector components are arranged in a cylindrical configuration, as shown in Fig.1, and are assembled in a pressure vessel maintained at one atmosphere. A particle traversing the apparatus passes through a uniform field of one Tesla produced by a thin $\left(4 \mathrm{~g} / \mathrm{cm}^{2}\right)$ superconducting coil and its trajectory is detected by a jet-type drift chamber (JET) and two inner drift chambers (IDC). The rigidity of the particle is determined by fitting these tracks using up to 28 hit points on each track. The maximum detectable rigidity (MDR) of this system is $200 \mathrm{GV}$. The time-of-flight (TOF) information is measured using scintillator hodoscopes located at the top and the bottom of the detector. The time resolution of the TOF system was 270 ps in the 1993 and 1994 fights, and improved to 110ps in the 1995 flight, and $70 \mathrm{ps}$ since 1997 . The $\mathrm{d} E / \mathrm{d} x$ information is measured by using the TOF counters and by the JET chamber.

Since BESS was intended to search for antiparticles, the trigger system was designed to detect negatively charged particles with higher than effciency than for positively charged particles while sampling a fraction of unbiased charged particles. The trigger consists of two levels, the T0 trigger and the $\mathrm{T} 1$ trigger. The $\mathrm{T} 0$ trigger, a basic instrument trigger, is provided by a simple coincidence between the top and the bottom TOF counters. It initiated digitization of various electronic modules and event building processes. As the T0 trigger rate of a few kilohertz is too high to record, the $\mathrm{T} 1$ trigger is adopted after the T0 trigger to discard the majority of positive low-energy particles. The T1 trigger was a hard-wired logic which determined the coarse track rigidity based on the IDC/TOF hit cell information. In order to determine the $\mathrm{T} 1$ trigger efficiency and measure the flux of positively charged particles, sampled T0 triggers with known sampling frequency or countdown (CD) were recorded irrespective of the $T 1$ trigger. The final recording rate was a few hundred hertz. We recorded all the triggered events on magnetic tapes.

\section{Analysis}

We have searched for antihelium in all recorded data. First, we applied the following criteria to select single track events:

1. There was only one hit in each layer of the 
TOF, allowing for one additional hit in the bottom layer.

2. One and only one of track with 10 or more hits should be found in the JET chamber.

3. The number of hits in the central region of the JET chamber expected from the trajectory was 16 or more.

Second, we required the following conditions to assure track-fitting quality and to remove possible scattering in the detector:

1. The number of hits used in the trajectory fitting $N_{r \phi-f i t} \geq 16$, and the reduced chisquare had to be less than 5 .

2. The number of hits used in the $z$-trajectory fitting $N_{z-\text { fit }} \geq 6$, and the reduced chisquare had to be less than 5 .

3. The track should be extrapolated in the $r-\phi$ plane to the TOF hit counter.

4. The extrapolation of the track should match the good IDC hit.

5. The number of "missing" hits which were expected from the trajectory but did not actually exist close to the expected position in JET chamber should be less than 4 .

Third, we selected $|Z|=2$ events with following conditions:

1. The velocity, $\beta$, should be consistent with downward going (anti) helium. Events outside the $\beta$ band were rejected as shown in Fig. 2. This selected the particles with $\mathrm{A} / \mathrm{Z}$ between $3 / 2$ and 2 .

2. The $\mathrm{d} E / \mathrm{d} x$ in the TOF and JET chamber should be consistent with $|Z|=2$ particles. Events in $\mathrm{d} E / \mathrm{d} x$ bands were selected as shown in Fig. 3.

All the above off-line selections were applied irrespective of the charge sign. Fig. 4 shows the $1 /$ rigidity distribution after above selections. No antihelium candidates were found in a rigidity region of 1-14 GV. The resultant upper limit for

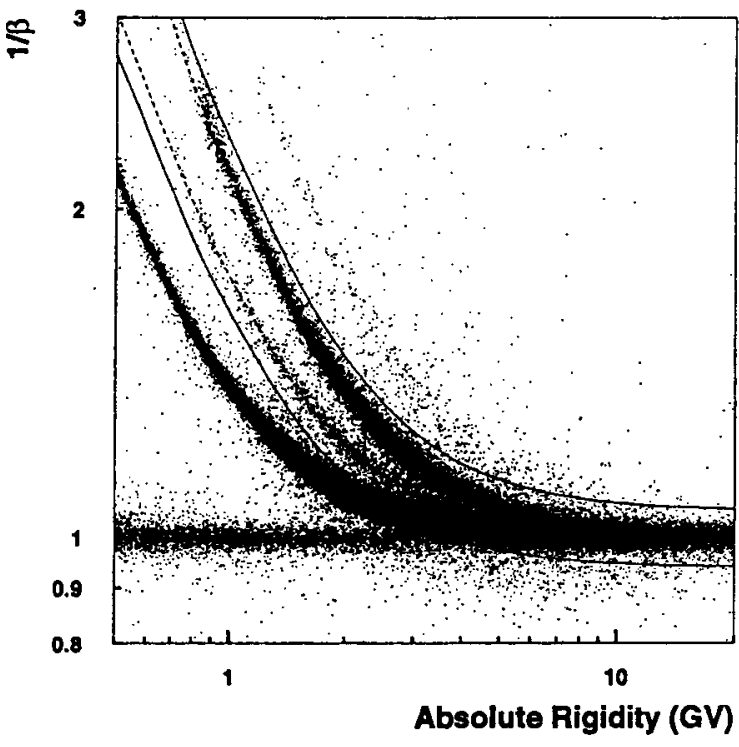

Figure 2. The $\beta^{-1}$ vs. absolute rigidity. Solid lines are applied for rejection and the dashed lines are the peak of ${ }^{3} \mathrm{He}$ and ${ }^{4} \mathrm{He}$.

the ratio of antihelium to helium in cosmic rays, $R_{\overline{\mathrm{He}} / \mathrm{He}}$, at the top of the atmosphere (TOA) is given by:

$R_{\overline{\mathrm{He}} / \mathrm{He}}=$

$\frac{\int N_{O b s, \overline{\mathrm{He}}} /\left(S \Omega \times \bar{\eta} \times \bar{\epsilon}_{s n g l} \times \bar{\epsilon}_{t r i g} \times \bar{\epsilon}_{d E / d x} \times \bar{\epsilon}_{\beta} \times \bar{\epsilon}_{T Q}\right) d E}{\int N_{O b s, \mathrm{He}} /\left(S \Omega \times \eta \times \epsilon_{s n g l} \times \epsilon_{t r i g} \times \epsilon_{d E / d x} \times \epsilon_{\beta} \times \epsilon_{T Q}\right) d E}$,

where the $N_{o b s}$ is the number of observed $\mathrm{He}(\overline{\mathrm{He}})$ events, the $S \Omega$ is the geometric acceptance of the BESS detector and does not depend on the sign of particle, $1-\eta(1-\bar{\eta})$ is the probability to lose $\mathrm{He}$ $(\overline{\mathrm{He}})$ by interactions with air, $\epsilon_{\text {sngl }}\left(\bar{\epsilon}_{\text {sngl }}\right)$ is the single track efficiency for $\mathrm{He}(\overline{\mathrm{He}}), \epsilon_{t r i g}\left(\bar{\epsilon}_{t r i g}\right)$ is the trigger efficiency for $\mathrm{He}(\overline{\mathrm{He}}), \epsilon_{d E / d x}\left(\bar{\epsilon}_{d E / d x}\right)$ is the $\mathrm{d} E / \mathrm{d} x$ cut efficiency for $\mathrm{He}(\overline{\mathrm{He}}), \epsilon_{\beta}\left(\bar{\epsilon}_{\beta}\right)$ is the $\beta$ cut efficiency for $\mathrm{He}(\overrightarrow{\mathrm{He}})$ and $\epsilon_{T Q}\left(\bar{\epsilon}_{T Q}\right)$ is the Track Quality Cut efficiency for $\mathrm{He}(\overline{\mathrm{He}})$. The numerator in eq. (1) is the number of anti- 

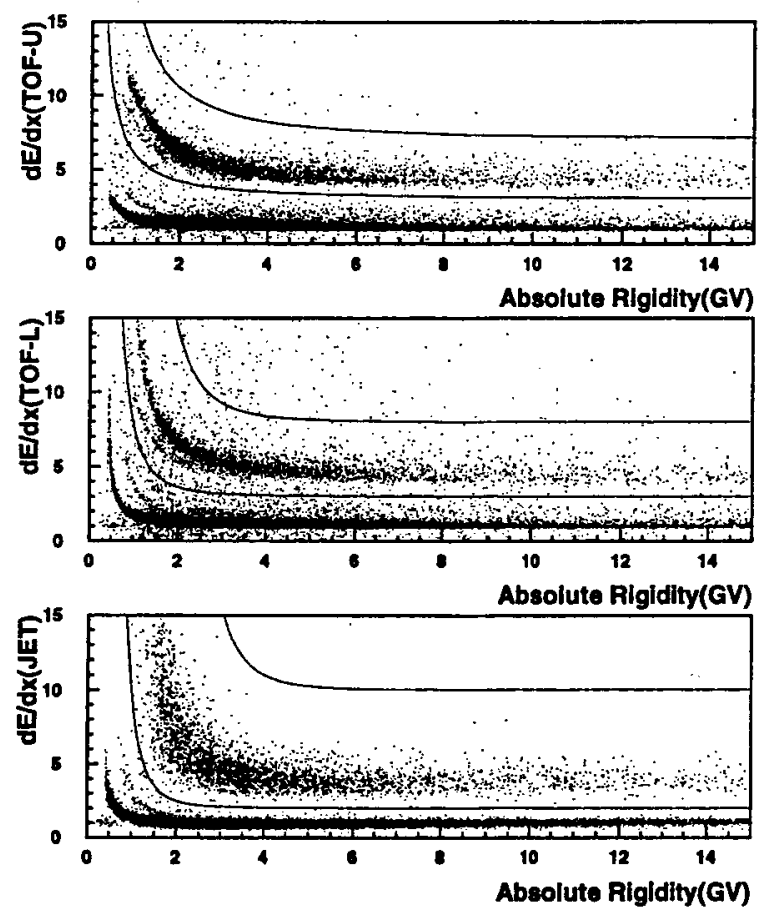

Figure 3. $\mathrm{d} E / \mathrm{d} x$ vs. absolute rigidity measured by top and bottom TOF and JET. The solid lines are applied for selection.

helium at the top of the atmosphere (TOA) and the denominator is the number of helium at the TOA.

The $S \Omega, \eta(\bar{\eta})$ and $\epsilon_{\text {sngl }}\left(\bar{\epsilon}_{\text {sngl }}\right)$ terms were evaluated by using the Monte Carlo simulation which was improved to incorporate the detailed descriptions of various interactions of helium nuclei and antihelium nuclei in the GEANT/GHEISHA code $[14,15]$. The cross sections for nuclear interactions of helium nuclei were determined by fitting the accelerator data, especially the cross sections for inelastic interactions in which ${ }^{4} \mathrm{He}$ goes to ${ }^{3} \mathrm{He}$, ${ }^{3} \mathrm{H},{ }^{2} \mathrm{H}$ and $\mathrm{H}$. The kinematics of the outgoing particle $\left({ }^{3} \mathrm{He},{ }^{3} \mathrm{H},{ }^{2} \mathrm{H}\right.$ and $\left.\mathrm{H}\right)$ were assumed to nearly equal the kinetic energy per nucleon and direction of the incoming ${ }^{4} \mathrm{He}$. We have not distinguished ${ }^{4} \mathrm{He}$ or ${ }^{3} \mathrm{He}$ in this analysis, so the in-

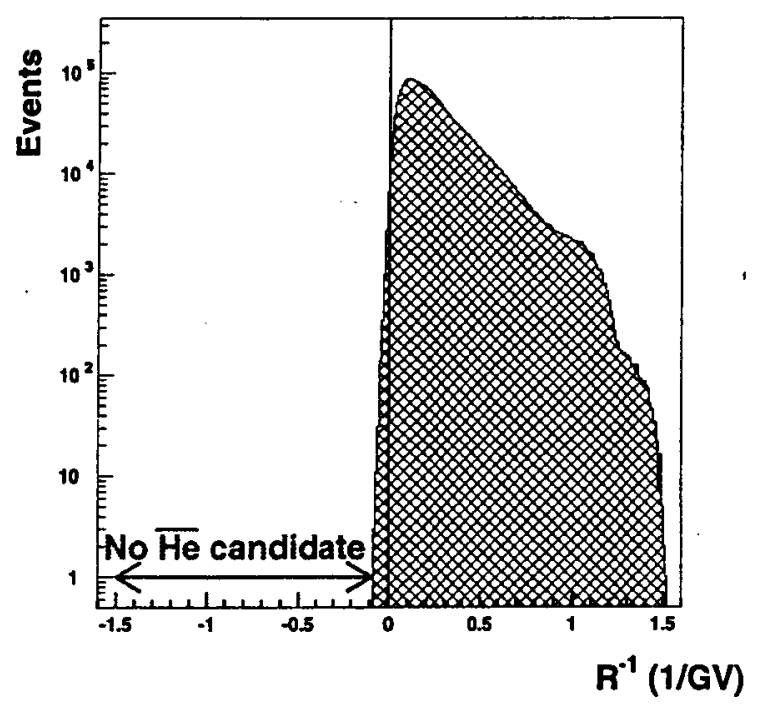

Figure 4. 1/R distribution of selected He events.

teraction $\left({ }^{4} \mathrm{He}\right.$ goes to $\left.{ }^{3} \mathrm{He}\right)$ was not considered as a loss of particles and did not affect the resultant efficiencies. There was no experimental data for nuclear interactions of antihelium nuclei, so we have assumed the following conditions to determine cross sections for the antihelium nuclei:

- The inelastic cross sections of antihelium obey the empirical model of hard spheres with overlaps [16], as described as follows, $\sigma\left(A_{i}, A_{t}\right) \propto\left(A_{i}^{1 / 3}+A_{t}^{1 / 3}-0.71 \times\left(A_{i}^{-1 / 3}+\right.\right.$ $\left.\left.A_{t}^{-1 / 3}\right)\right)^{2}$,

where $\sigma\left(A_{i}, A_{t}\right)$ is the cross section of an incident particle with atomic weight $A_{i}$ to a target with atomic weight $A_{t}$.

- Antihelium is always fragmented when an inelastic interaction occurs.

- The elastic cross sections of antihelium are the same as those of helium.

While the geometric acceptance of the BESS detector $(S \Omega)$ depended slightly on the energy, it turned out to be almost constant in the relevant energy region. The $\eta(\bar{\eta})$ were over 0.8 and 
$\epsilon_{s n g l}\left(\bar{\epsilon}_{\text {sngl }}\right)$ were over 0.5 in the relevant energy region. The $\epsilon_{d E / d x}, \epsilon_{\beta}$ and $\epsilon_{T Q}$ were derived from the real data. The $\mathrm{d} E / \mathrm{d} x$ selection efficiency and $\beta$ cut efficiency for He were higher than 99 $\%$. There were no antihelium events. However, the off-line selections we have applied do not depend on the charge sign, so we have assumed the $\bar{\epsilon}_{d E / d x}, \bar{\epsilon}_{\beta}$ and $\bar{\epsilon}_{T Q}$ were the same as $\epsilon_{d E / d x}, \epsilon_{\beta}$ and $\epsilon_{T Q}$, respectively. The $\epsilon_{t i r g}$ depended on the sampling frequency of the T0 trigger, and is $1 / 40$ for the ' 93 flight, $1 / 15$ for the first half and 1/30 for the last half of the '94 flight, 1/20 for the '95 flight, $1 / 25$ for the ' 97 to ' 99 flights, and $1 / 30$ for the '00 flight. The $\left(\bar{\epsilon}_{\text {trig }}\right)$ was determined by using CD sample events. The upper bound of the integral in Eq. 1 was determined by the edge of the spillover in the negative rigidity region and the lower bound of integral was determined to be $0.18 \mathrm{GeV} / \mathrm{n}$ at TOA (a rigidity of $1 \mathrm{GV}$ at the center of the detector). All efficiencies were high in this region as shown Fig. 5.

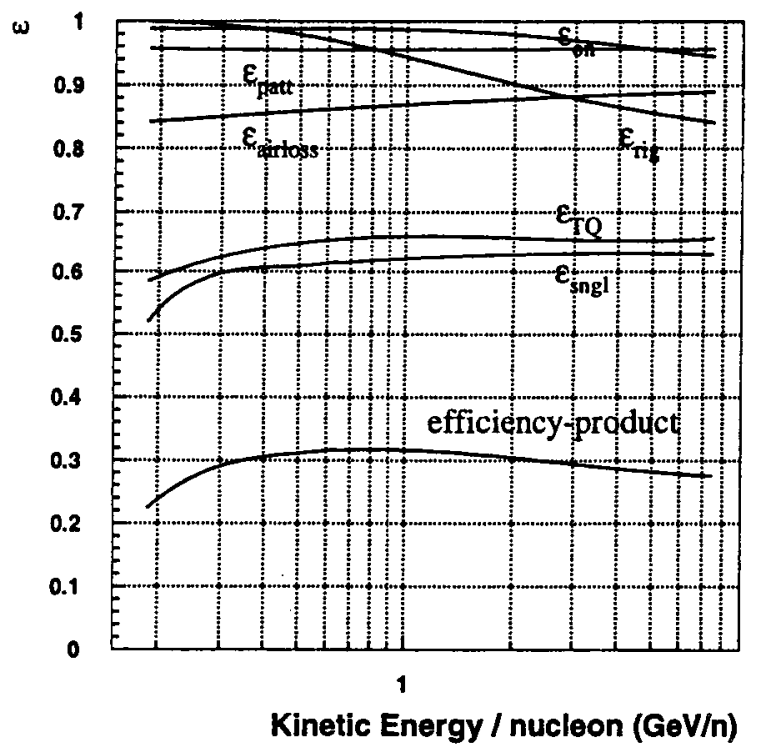

Figure 5. Selection efficiencies and their product applied for 1998-flight data.
Since we found no antihelium candidate with rigidity below $14 \mathrm{GV}$, only an upper limit can be set by using Eq. 1 in this rigidity region. We take 3.1 as the number of antiheliums $(\overline{\mathrm{He}})$ for the calculation of the $95 \%$ confidence level in the upper limit. To integrate the numerator in Eq. 1 , we must assume the energy spectrum of antihelium because the efficiencies are a function of energy. In order to obtain the most conservative limit, we used the minimum value of the antihelium efficiency-product in the energy region, to be 0.22 , to integrate the numerator.

We can also give an upper limit with the modeldependent assumption that the incident $\overline{\mathrm{He}}$ energy spectrum has a same shape as the He spectrum to compare our results with other experiments. Under this assumption, Eq. 1 becomes

$R_{\overline{\mathrm{He}} / \mathrm{He}}<$

$\int \frac{3.1 \mathrm{~d} E}{N_{\text {Obs, He }} \times \bar{\eta} \times \bar{\epsilon}_{s n g l} \times \bar{\epsilon}_{t r i g} /\left(\eta \times \epsilon_{s n g l} \times \epsilon_{t r i g}\right)}$.

\section{Results and conclusion}

We have searched for antihelium nuclei in cosmic rays using the BESS spectrometer. We reanalysed all the data collected during 1993-2000 with a common analysis procedure. This result is shown in Fig. 6 and is compared with previous limits $[6,5,4,7-9,1-3]$. The total number of helium nuclei observed by BESS 1993-2000 was $>$ $6.6 \times 10^{6}$ in a rigidity region of $1 \mathrm{GV}$ through 14 GV. No antihelium candidate was found in this rigidity region. The resultant $95 \%$ confidence level upper limit on the $\overline{\mathrm{He}} / \mathrm{He}$ flux ratio at the top of the atmosphere in the rigidity range from 1 to $14 \mathrm{GV}$ was $8.6 \times 10^{-7}$ with a model independent assumption and $6.8 \times 10^{-7}$ with a model dependent assumption that the $\overline{\mathrm{He}}$ energy spectrum coincided with the He spectrum.

We thank the NASA/GSFC/WFF Balloon office and the National Scientific Balloon Facility (NSBF) for the balloon expedition, and KEK and ISAS for various supports. This work was supported by Grant-in-Aid for Scientific Research, Monbusho and by Heiwa Nakajima Foundation in Japan; and by NASA in the USA. A part of 
analysis was performed using the computing facilities at ICEPP, the University of Tokyo.

\section{REFERENCES}

1. Ormes, J.F. et al. Astrophys. J. 482 (1997) 187-190.

2. Saeki, T. et al., Phys. Lett. B 422 (1998) 319324.

3. Alcaraz, J. et al., Phys. Lett. B 461 (1999) 387-396.

4. Aizu, H. et al., Phys. Rev. 121 (1961) 1206.

5. Evenson, P., Astrophys. J. 176 (1972) 797808.

6. Smoot, G.F. et al., Phys. Rev. Lett. 35 (1975) 258.

7. Badhwar, G.D., Golden, R.L. et al., Nature 274 (1978) 137.

8. Golden R.L. et al., Astrophys. J. 479 (1997) 992-996.

9. Buffington A. et al., Astrophys. J. 248 (1981) 1179.

10. Ajima, Y. et al. Nucl. Instr. and Meth. A 443 (2000) 71-100.

11. Asaoka, Y. et al. Nucl. Instr. and Meth. A 416 (1998) 236-242.

12. Shikaze, Y. et al. Nucl. Instr. and Meth. A 455 (2000) 596-606.

13. Yamamoto, A. et al. IEEE Trans. Magn. (1988) 1421-.

14. Matsumoto, H., Doctoral thesis of Kobe University, (1999) 1135-1142.

15. Sanuki, T. et al., Astrophys. J., 545 (2000) 1135-1142.

16. Bradt, H.L. and Peters, B., Phys. Rev.77 (1950) 54.

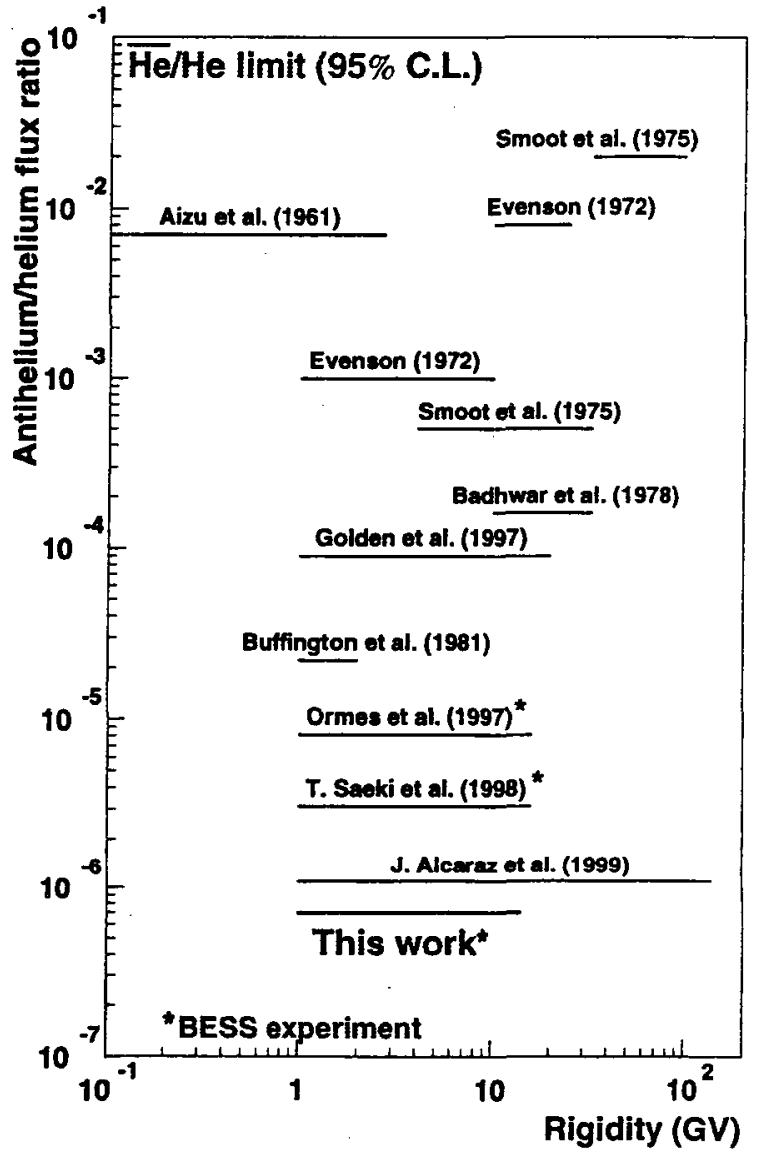

Figure 6. New upper limit of $\overline{\mathrm{He}} / \mathrm{He}$ obtained in this work shown with previous BESS results(BESS 1993-1995 and 1997-2000), and with other experiment results. 